\title{
Measurement on Surface Temperature Error of Fiber Bragg Grating Temperature Sensor
}

\author{
Yi Xie, Guofeng Ma, Yuan Cheng \\ Wuhan University of technology, Wuhan, 430010, China
}

Keywords:Fiber Bragg grating, temperature sensor, measurement error, compensation.

\begin{abstract}
Fiber Bragg grating temperature sensor has the characteristics of strong anti-jamming capability, distributed measurement which has a good application prospect in all kinds of industrial fields. But in the actual measurement of surface temperature, error caused by temperature gradient caused of the FBG is often ignored. In this paper, researches on finite element and experiments were proposed. The influence factor, and the linear compensation of FBG temperature sensor were found to compensate the property of FBG temperature sensor. The results showed the measurement error ranged from 4 degree centigrade to 14 degree centigrade, which can improve the measurement accuracy of the temperature sensor.
\end{abstract}

\section{Introduction}

Fiber Bragg grating (FBG) is emerging since the 1990s which has the advantage of strong anti-interference, corrosion resistance, light quality, long service lifeandno loss of connection, which can realize more advantages of distributed measurement and has a broad application prospect in the field of optical sensing [2, 6]. Fiber Bragg grating sensor can be used for temperature, pressure, strain, which has important application value in modern industrial production. In order to improve the convenience of installation and the resolution of the measurement, many scholars in researches on the structure and sensitization principle of fiber Bragg grating [3, 4], but few research entered on its measurement error. Mingyao Liu [5] and others stacked FBG on lathe spindle which ignored the FBG measurement error. GanWeiBing [1] used the encapsulated fiber Bragg grating temperature sensor in the water and analysed the temperature measurement error, but the surface temperature measurement error of fiber Bragg grating was not studied. This paper proposed the theory analysis and experiments on the copper surface temperature measurement error and compensates the error of the sensor, improve the measurement precision of the fiber Bragg grating. At last, it provides the basis for fiber Bragg grating in FBG temperature measurement.

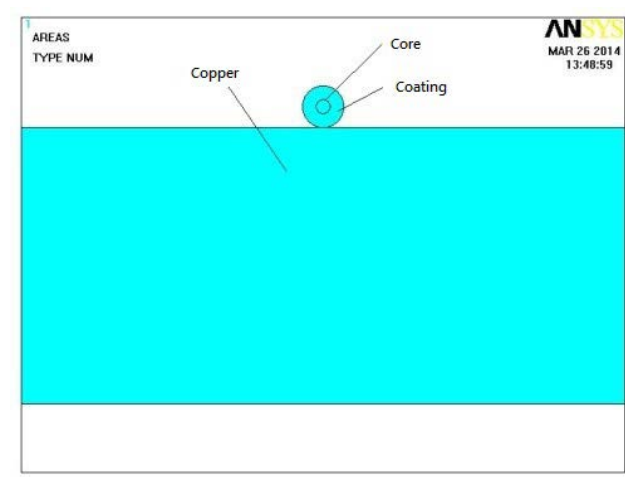

Fig. 1 the model of surface temperature measurement setup

\section{Theory}

\subsection{Analysis of the surface measurement error based on the finite element analysis}

For the general surface temperature measurement, it can be supposed the object has the uniform temperature, fiber Bragg grating is closely placed on the surface under the situation which is shown in Fig.1. When the heat steady state thermal analysis is taken into consideration, set the heat conductivity coefficient of the copper and fiber core $401,0.046,0.77 \mathrm{~W} / \mathrm{m} *^{\circ} \mathrm{C}$, and the air convection 
coefficient is $20 \mathrm{~W} / \mathrm{m} *{ }^{\circ} \mathrm{C}$. When the temperature of the copper surface ranges from $40{ }^{\circ} \mathrm{C}$ to $90{ }^{\circ} \mathrm{C}$, after steady-state transient thermal analys by ANSYS, the temperature difference between the fiber core and the copper is shown as Fig.2. It can be concluded the difference and copper temperature has a good linear relation.

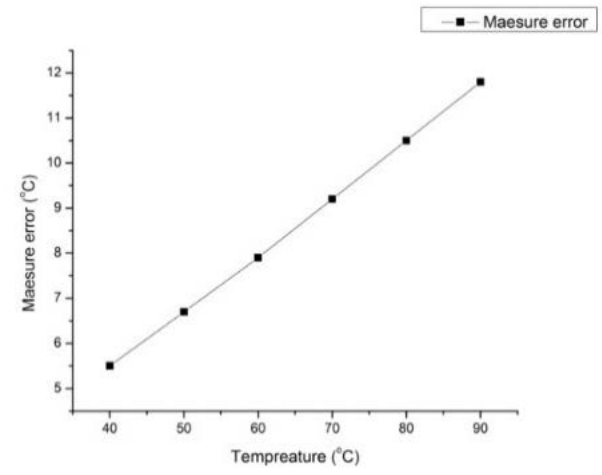

Fig. 2 the relationship between fiber core temperature and the copper surface temperature

\subsection{Compensation principle}

According to the principle of fiber Bragg grating coupling, when a beam of light into the uniform fiber grating, the Bragg conditions of light wave will be reflected, the reflection peak wavelength to meet:

$$
\lambda_{B}=2 \mathrm{n}_{\text {eff }} \Lambda(1)
$$

Type: $\mathrm{n}_{\text {eff }}$ for the fiber core refractive index effectively; The grating stripe cycle.

As you can see, from the type $n_{\text {eff }}$ and $\Lambda$ the change will cause the change of the reflection wavelength.When the fiber Bragg grating external environment changes, will cause the corresponding change, leading to drift. And strain (or stress) and temperature is directly affect the quantity of Bragg grating wavelength, if the assumption that the temperature and strain caused by the center wavelength were independent of each other, ignore the centre wavelength sensitivity coefficient caused by impact, then the temperature and the strain with the wavelength change under the available type said:

$$
\Delta \lambda_{B}=\alpha_{E} \bullet \varepsilon+\alpha_{T} \bullet \Delta \mathrm{T}
$$

Type: $\alpha_{E}$ for the sensitivity coefficient of fiber Bragg grating strain; $\alpha_{T}$ as the sensitivity coefficient of fiber Bragg grating temperature; As the temperature variation; $\varepsilon$ for strain.

In this experiment, the fiber Bragg grating to single-ended fixed, so you can ignore the influence of the strain, the wavelength is only produced by the temperature change can be represented as:

$$
\Delta \lambda_{B}=\alpha_{T} \bullet \Delta T
$$

By the type, the quantity of the center of the fiber Bragg grating wavelength drift temperature variation is linear relationship;Is the center of the fiber Bragg grating wavelength has a linear relation with temperature, can be represented as:

$$
\lambda_{B}=\mathrm{k}_{1} \bullet T+\lambda_{0}(4)
$$

Type: $\mathrm{k}_{1}$ and $\lambda_{0}$ for related coefficient.

Using fiber Bragg grating measuring error of surface temperature, based on the finite element simulation of steady state thermal analysis, it is concluded that the measurement error and to be a linear relationship between the actual temperature measurement, can be represented as:

$$
\Delta T=T_{0}-T=\mathrm{k}_{2} \bullet T_{0}+\Delta T_{0}
$$

Type: $\Delta T$ as the measurement error; $T_{0}$ by measuring the actual temperature; $T$ for fiber Bragg grating temperature have been measured, $\mathrm{k}_{2}$ and $\Delta T_{0}$ for related coefficient

Through the type (5) compensate type (4), compensation formula. Can be represented as:

$$
\lambda_{B}=\mathrm{k}_{1} \bullet\left(1-\mathrm{k}_{2}\right) \bullet T_{0}-\mathrm{k}_{1} \bullet \Delta T_{0}+\lambda_{0}(6)
$$


Through the type to calculate the temperature value will increase by fiber bragg grating temperature sensor error of the measured surface temperature value, improve the accuracy of measurement.

\section{The experiment}

\subsection{Copper surface temperature measurement device:}

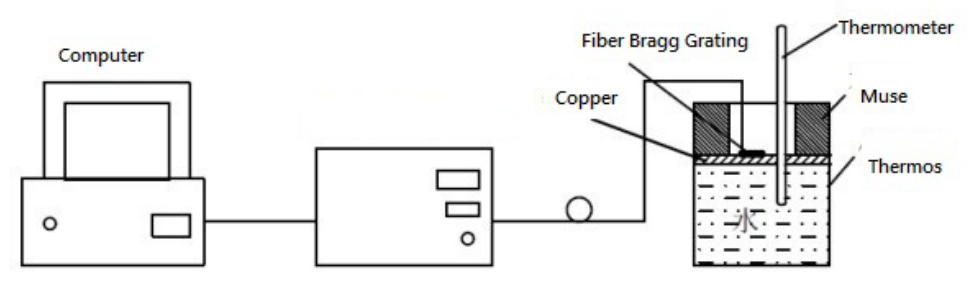

Fig. 3 copper surface temperature measuring device

Fig.1 measurement device for copper surface, fiber Bragg grating temperature sensor fixed on the copper sheet end, via a jumper wire connection on FBG demodulation devices, demodulation devices through the cable connection on the computer, can be read out the centre of the fiber Bragg grating wavelength values, calculated temperature measured by fiber Bragg grating can be obtained. Up and down the water in the thermos been fully stirring, water temperature difference is less than $0.1{ }^{\circ} \mathrm{C}$ and the copper contact with the water, so the thermometer measured numerical approximation for the temperature of copper. Through the thermometer measured temperature value and calculate the temperature value, can be concluded that optical fiber grating temperature sensor error value of the copper surface temperature measurement.

\subsection{The experimental measurement and results:}

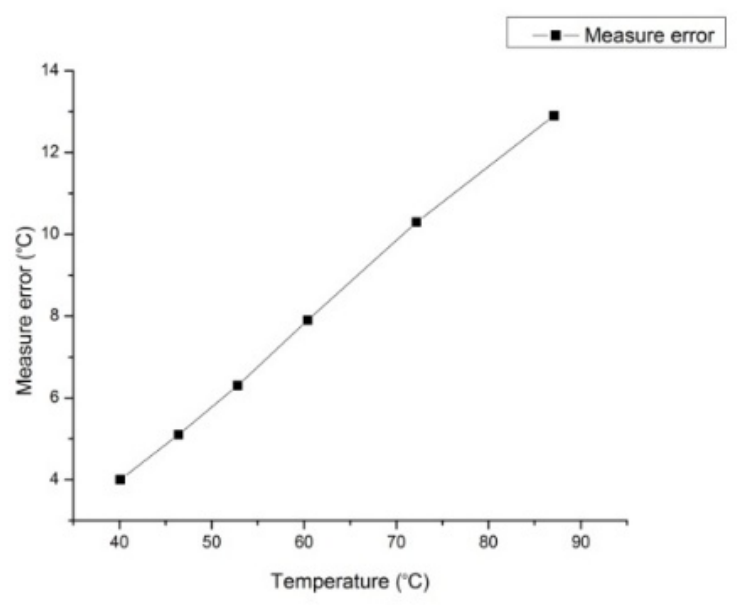

Fig.4 measurement error of measured surface temperature and diagram

Copper surface temperature rise from $40^{\circ} \mathrm{C} 90^{\circ} \mathrm{C}$, the fiber Bragg grating temperature sensor measurement error as shown in fig.4.You can see from figure measurement error had good linear relationship with the actual temperature measurement, the correlation coefficient of 0.99 above, the temperature measurement error and the actual temperature of the linear fitting equation, the steady state thermal analysis with finite element results.

Using the linear fitting equation of centre wavelength of $1306 \mathrm{~nm}$ fiber Bragg grating temperature sensor measurement error of the copper surface temperature error compensation, its compensation before and after the compensation of the measurement error is shown in figure 5.Can see from figure, when copper changes from $40^{\circ} \mathrm{C}$ temperature is $90^{\circ} \mathrm{C}$, the compensation in front of the measurement error of $4^{\circ} \mathrm{C}$ and $14^{\circ} \mathrm{C}$, but the measurement error is within $1{ }^{\circ} \mathrm{C}$ after compensation, to improve the 
accuracy of measurement, and the linear relation between the measured temperature measurement error and the repeatability is good.

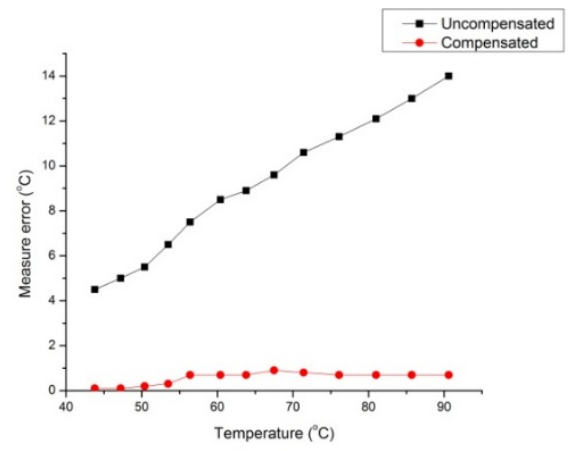

Fig. 5 Compensation and compensation of measurement error

\section{Conclusion}

Fiber Bragg grating temperature sensor measurement error of the copper surface temperature measured, the measurement error has a linear relation with the actual temperature measurement, through the linear relationship between the measurement error can be linear compensation. When the copper surface temperature of $40{ }^{\circ} \mathrm{C}$ up to $90^{\circ} \mathrm{C}$, compensation, its measurement error is $4{ }^{\circ} \mathrm{C}$ and $14{ }^{\circ} \mathrm{C}$, but after compensating the error of measurement within $1{ }^{\circ} \mathrm{C}$, so compensation of fiber Bragg grating temperature sensor in measuring surface temperature more accurate, higher precision, for fiber grating on the surface temperature measurement.

\section{References:}

[1] GAN Weibing, WANG Lixin , ZHANG Cui. Distributed Optical Fiber Grating Technology Applied in Cable Temperature Measurement [J].Laser and Infrared, 2011, 41(5), 578-581.

[2] HE Huiling, ZHAO Chunmei , CHEN Dan ,et al..Present Status of Optic Sensors [J].Laser and Optoelectronics Progress, 2004, 41 (3):39-41.

[3] He Wei , Xu Xiandong , Jiang Desheng.High-Sensitivity Fiber Bragg Grating Temperature Sensor with Ploymer Jacket and Its Low-Temperature Characteristic[J].Acta Optica Sinica , 2004,24(10),1316-1319.

[4] LIU Qinpeng, Qiao Xueguang, JIA Zhenan,et al.. Research and Development of Fiber Bragg Grating Enhancing Sensitivity [J].Transducer and Microsystem Technologies, 2006, 25(4), 5-7.

[5] Mingyao Liu,Erlong Zhang,Zude Zhou,Yi Liu."Measurement of Temperature Field for the Spindle of Machine Tool Based on Optical Fiber Bragg Grating Sensors,”Advances in Mechanical Engineering, 2013.

[6] TANG Wei,SHI Yi Kai. Study on applications of fiber Bragg grating sensing technique [J]. Opt. Precision Eng. 2002, 10(1):79-83. 